\title{
Optical mapping with the ARIANE HROV at IFREMER : the MATISSE processing tool
}

\author{
Arnaubec Aurelien ${ }^{1,{ }^{*}}$, Opderbecke Jan ${ }^{1}$, Allais Anne-Gaelle ${ }^{1}$, Brignone Lorenzo ${ }^{1}$ \\ 1 IFREMER, Underwater Syst Dept, BP 330, F-83507 La Seyne Sur Mer, France. \\ * Corresponding author : Aurélien Arnaubec, email address : aurelien.arnaubec@ifremer.fr
}

\begin{abstract}
:
In the scope of the development of IFREMERs hybrid underwater vehicle named 'HROV Ariane', seafloor mapping with optical imagery sensors is a major function which has motivated the development of a software processing tool for semi-automatic, on-line or off-line, 2D and 3D optical mapping. We present in this paper development results for a complete software framework intending to make state-of-the-art and future algorithms available for routine operation. The aim is to provide a software tool able to produce 2D/3D maps from high resolution image sequences merged with navigation data in a robust and simplified process without requiring the presence of a specialist controlling the computation process. The 2D mapping technique aims to merge many thousands of multiple view, small footprint images, into a single geo-referenced mosaic that accounts for image and navigation fusion. The 3D mapping is more suitable for smaller areas and aims to reconstruct geo-referenced and scaled 3D models of sea-floor scenes from multiple image frames. Algorithms integrated to the Matisse tool have been extensively tested on several datasets and in real world trials, ensuring robust performance in numerous environmental scenarios.
\end{abstract}




\section{INTRODUCTION}

Autonomous optical mapping is one of the prime functions of IFREMERs hybrid underwater vehicle (HROV). The system is specially designed for surveying underwater canyons and cliffs. Canyons of the French Mediterranean coast typically stretch from the continental shelf at roughly $200 \mathrm{~m}$ depth, to the abyssal planes at more than $2000 \mathrm{~m}$. The canyons are precious sites, with unique biological activity and biodiversity; they are targeted by integrated oceanographic monitoring and protection projects at national and European levels.

Optical mapping, consisting of large scale geo-referenced mosaicking in 2D [1]-[8] and in 3D [9]-[11], can be of prime interest for studying canyons sites and hence, is an important function of the HROV system. With the developed algorithms a first optical overview of the sea floor can be obtained by real-time processing, producing geo-localized and scaled mosaics of limited extent, the successive mosaic blocks being successively loaded and visualized in a GIS-tool (the file format being standard GeoTiff tiles). A more precise technique is also proposed in post-processing.

In order to offer a simplified use of the mosaicking algorithms in an operational environment, we have designed a software suite called Matisse. The various algorithms are integrated in the form of separate modules, which can be logically connected in order to define a complete mosaicking process.

The first section gives a brief overview of the HROV optical survey capabilities and the second section presents a pre-processing method to correct images for non uniform illumination.

The third section treats about 2D optical mosaicking technique, that has been optimized to process very large scale mosaics of many thousands images. It is thus useful to obtain a global representation of the sea floor but does not provide any information of the vertical structure of the scene.

To overcome this limitation, we present in the fourth section, 3D reconstruction algorithm using a single camera and images taken from successive locations. This algorithm is based on "structure from motion" techniques, and can provide an highly precise bathymetry at small scale and hence is a good complement to multi-beam bathymetry.

Finally, we present how we integrated those processing techniques in an end-user oriented tool that can be used to process data without strong image processing expertise.

\section{IMAGE ACQUISITION}

The Ariane HROV is a new concept of underwater vehicle developed at IFREMER (see vehicle on dive in Fig 1). The $\mathrm{H}$ stands for hybrid, meaning that the HROV can both work as an AUV or an ROV. In ROV mode, the only link between the vehicle and the surface is a reusable optical fiber. In case the fiber break the vehicle is able to continue or abort its mission depending on the type of mission.

Concerning the optical payloads, the HROV is equipped with multiple HD cameras and a high resolution (24Mpix) Nikon DSLR still camera. One important task of the HROV concern the autonomous optical acquisition of canyon sites. For this, the vehicle is equipped with state of the art navigation system and a frontal dvl.

This enable to perform optical surveys with precise georeferencing of the optical data, which is of prime importance for the mapping algorithms presented in the following. 


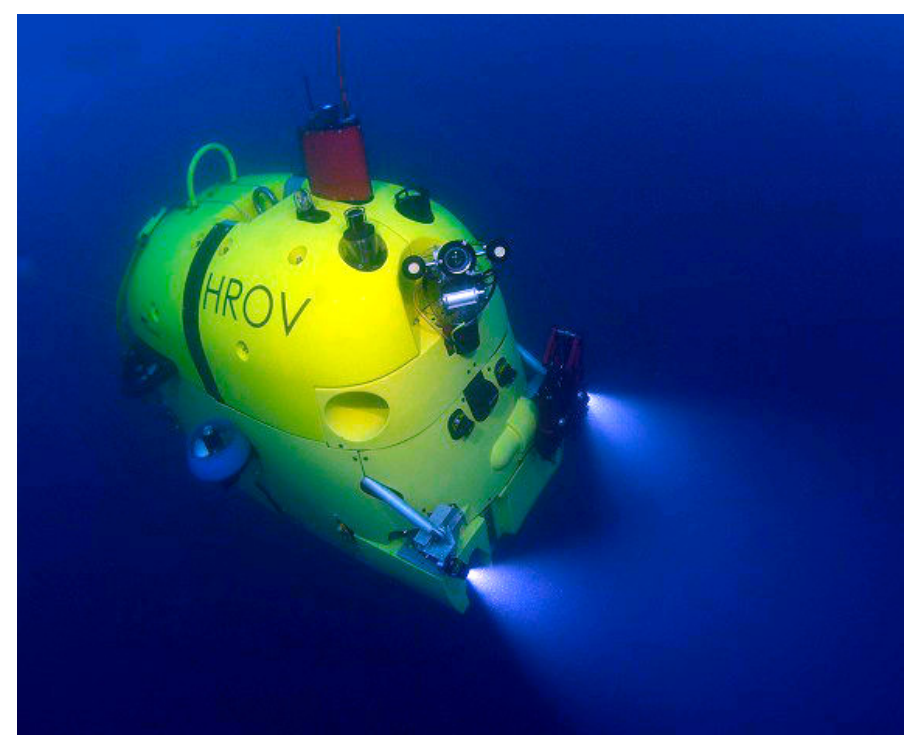

Fig. 1. IFREMER Ariane HROV during a dive.

\section{IMAGE PREPROCESSING}

Underwater optical images can suffer from a lot of disturbances such as non-uniform illumination, color dependent attenuation, marine snow. Pre-processing is hence a critical step for final result quality. Algorithms have been developed for enhancing the images in the pre-processing phase [1], [2]. We present in this section a model based illumination correction.

Deep water optical imaging requires artificial illumination of the scene as light is highly attenuated in water and after a few tens of meter sunlight is not enough. However artificial light cannot be optimized to lighten uniformly the scene observed by the camera plane for any altitude and images often present non uniform illumination pattern disturbance as the on the image of Fig 2. Before any feature extraction or image analysis, deep sea optical images have to be compensated for non uniform illumination.
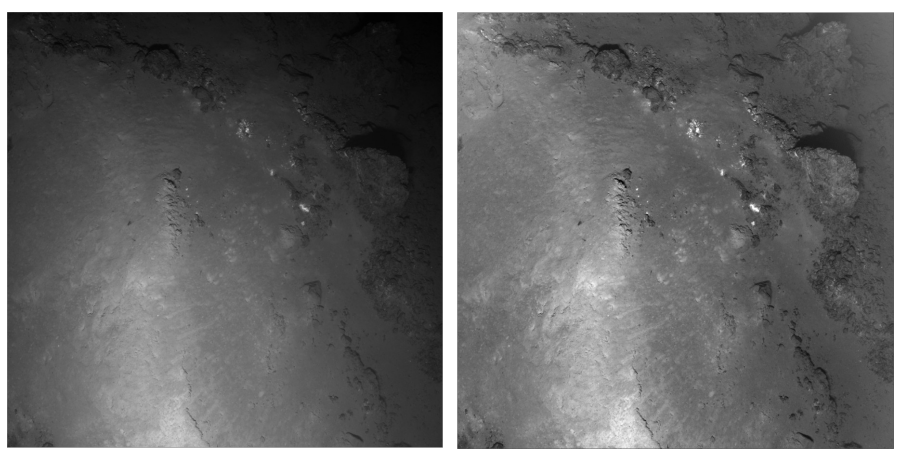

Fig. 2. On the left we present an example of deep sea image obtained with IFREMER Victor 6000 ROV high sensitivity black and white still camera. One can see the strong non uniformity of the illumination. On the right we present the same image for which we applied the proposed illumination correction.

Our method uses successive images acquired in quite the same illumination conditions and hence only information from the seafloor is changing along the different successive frames.

To remove information from the seafloor and only keep the illumination pattern, a sliding mean or median filter is applied. Some algorithms from the literature [1], [2] use the resulting image as an estimation to restore images as if they were uniformly illuminated. The issue is that if the windows size is too small, the estimation is noisy and while if too big the method is not adapted to changing floor slope and distance. The solution we propose uses a small window with model fitting.

We model the spot as a camera emitting light and the floor as a plane. It hence enables to describe the projection from the spot to the plane and then back to the camera as an homography transformation. We also suppose that the spot pattern $I_{s}(x, y)$ is a paraboloid of equation:

$$
I_{s}(x, y)=\alpha x^{2}+\beta y^{2}+I_{0}
$$

where $x$ and $y$ are the euclidean coordinates in the spot plane, $\alpha>0, \beta>0$ and $I_{0}>0$.

The Fig 3 gives a representation of the acquisition configuration. Under these conditions it can be shown that the equation of the spot pattern in the camera plane $I_{c}\left(x^{\prime}, y^{\prime}\right)$ is a third order polynomial of the form:

$$
\begin{aligned}
I_{c}\left(x^{\prime}, y^{\prime}\right)= & a x^{\prime 3}+b y^{\prime 3}+c x^{\prime} y^{\prime 2}+d x^{\prime 2} y^{\prime} \\
& +e x^{\prime 2}+f y^{\prime 2}+g x^{\prime} y^{\prime}+h x^{\prime}+i y^{\prime}+j
\end{aligned}
$$

where $x^{\prime}$ and $y^{\prime}$ are the euclidean coordinates in the camera plane and coefficients have non linear constraints.

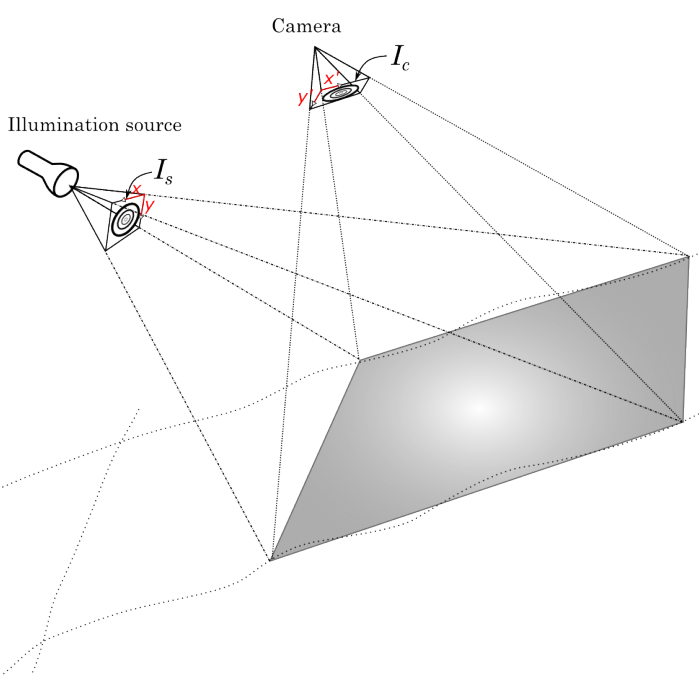

Fig. 3. This figure present a schematic representation of the proposed illumination model. The illumination source projects its light pattern on the sea floor plane, then the light is backscattered toward the camera plane.

For simplicity and computing speed we solve this problem using linear solver with the constraint that all fitting points have positive intensity (hence not accounting for non linear constraints that would involve more complex solver, convergence and speed issues). We then correct the image 
using this estimation.

An example of result is presented on the Figure 2, on which almost all intensity variations have been removed. This procedure has been tested on several of our dataset and is now considered as a systematic pre-processing, applied before any mosaicking technique presented in the following.

Optical images and videos are of prime interest for the analysis of the deep underwater environment which is not accessible for human intervention. However, the limited visibility imposes taking images close to the sea-floor and having a small footprint for each image. A global view of the scene is hence only possible with the construction of a map with multiple images. Every mosaicking technique uses the redundancy in successive images or within a video flux to put all pieces together and build the global view.

\section{2D MAPPING}

The first step in mosaicking consists in feature detection and matching. The matching has to be accurate robust and fast. We chose the most widely used SIFT (Scale Invariant feature transform) algorithm low99, justified by its strong robustness and accuracy, and speed given by GPU implementation. A first matching is obtained with the SIFT and then outliers are removed with the classical RANSAC (RANdom SAmple Consensus) robust estimation method with homography transformation model.

In order to avoid drift and scale the mapping, image matching information is merged with navigation through bundle adjustment. The developed method is similar to [3] except that cost function weight are affected according to image and navigation data standard deviation and re-projection errors are minimized in the mosaicking plane rather than the image plane.

The output of the bundle adjustment is optimized image positions. With this information the mosaic can be drawn and georeferenced. This is done in our case with state of the art seaming and blending techniques of [15], [16].

An example of result is presented in Fig 4. This has been first applied to post-processing mosaicking but the gain in time brought by GPU architecture enables us to evaluate real-time feasibility.

Real-time mosaicking has already been explored before at IFREMER with the MATISSE software operating with RMR (Robust Multi-Resolution) method [6]. The main drawback of this method is the need of a very high overlap between two successive images, that has been removed using sift features detection and matching. The real-time sift algorithm is based on the same principle but without the bundle adjustment. The test we did with this algorithm enabled us to mosaic 720p HD video sources in real-time.

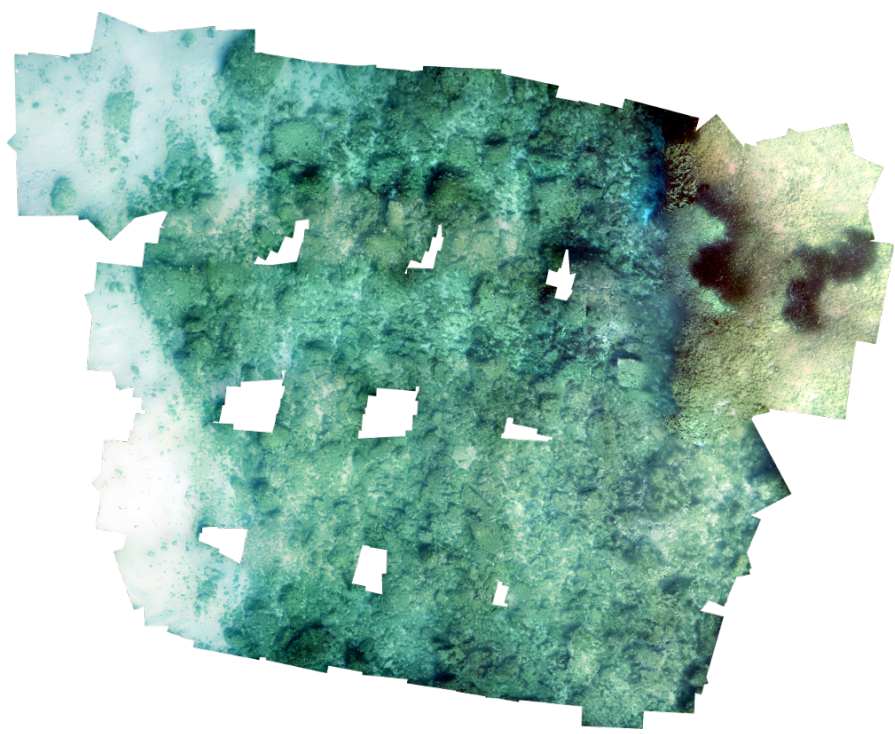

Fig. 4. This Figure presents an example of 2D mapping obtained with the method described in this paper. This color mosaic benefits from the fact that the same areas are visited multiple times to improve images localization.

\section{3D MAPPING}

One important mission of the HROV consists in surveying underwater canyons and cliffs, which means we will have to map scene with strong relief. Any 2D mosaicking technique map a projection of the scene on a plane and is hence not adapted to highly $3 \mathrm{D}$ scene. To overcome this limitation we evaluated the potential of $3 \mathrm{D}$ reconstruction techniques.

This topic has been extensively studied in the absence of the water medium and many techniques exist in the literature [9]-[13] One of the most promising family of techniques is "structure from motion" (SFM) techniques, based on multi-view geometry and images matching. It enables the reconstruction of $3 \mathrm{D}$ points from the scene and camera position from where the images were taken.

The feature detection and matching is the same as for 2D mapping except that the RANSAC model is based on the fundamental matrix. Then the first step consists in bundle adjustment using only image (no navigation data) for solving SFM problem which estimates 3D points and cameras position up to a scale ambiguity.

After the bundle adjustment, at most all 3D points detected by sift algorithm are reconstructed, called sparse reconstruction. Then, to increase the number of reconstructed points we use the dense matching method described in [17]. This method supposes that a few features points have been detected (here the Sift points) and that the position of the cameras are known (estimated during bundle adjustment). The dense matching is correlation based and hence enables to highly increase the number of reconstructed points. With this method, the orientation of the underlying surface is also estimated for each reconstructed point. 
At this stage, the cameras position and the dense cloud of points have been estimated up to a given scale and with a position and orientation relative to the first camera. In order to geo-reference those data and make measurements, the absolute position and scale have to be estimated. To do so, we estimate all those parameters by fitting the structure from motion cameras position to the ones measured by the navigation system in a least square sense. After this, the scene is metric and hence allows measurements in the reconstructed scene.

As the final step, we then reconstruct a surface (mesh) from the dense cloud point. We solve this problem using the poisson surface reconstruction algorithm described in [18]. An example of result is illustrated on Fig 5, using images obtained from a Nautile dive using the exact replica of the DSLR still camera mounted on the HROV. The DSLR high resolution (24Mpix) leads to a very dense $3 \mathrm{D}$ reconstruction.

\section{OPERATIONAL SOFTWARE INTEGRATION}

The HROV has been developed as an operational vehicle keeping in mind that data exploitation is mainly done by biologists and geologists. The same applies to optical mapping. We hence developed an end-user oriented software called Matisse, to integrate all the previously described algorithms.

We chose to divide this software in two part:

- An independent software for data processing and previsualization.

- A plug-in tool within QGIS to import and analyse processed data.

The independent software is based on a highly modular architecture designed for a general-user -non-expert approach. The software has a C++ API to develop imaging algorithms as plug-ins that can be dynamically loaded in Matisse. In expert mode (see Fig 7), the user defines an assembly of algorithms, affect expert parameters values and save the whole as a processing chain. The different blocks of the processing chain (each being dll plugins) can exchange any type of data in a completely generic way. This ensure that even if the algorithms change the way they handle data, the software will still be able to integrate them in a processing chain. Both real-time and post-processing work flow are available, which implies that the acquisition can also be done within Matisse.

Then, the end-user which does not need any detailed knowledge in how the algorithms work can apply this processing chain to any dataset (see Fig 6), through the notion of Job. He has the right to change some of the algorithms parameters which are defined as easily understandable by the expert. A Job is the instantiation of a processing chain on a given dataset with user chosen parameters. A job history is available in the user view to keep a trace of the processed datasets.

After the Job processing, a basic visualization of the result is available within the software. This visualization is based on the QGIS library and is optimized to handle large area images.

The user then import the result in QGIS using Matisse plug-in for advanced visualization and analysis (see Fig 8). With this choice the user benefits from all the measurement tools already implemented in QGIS. The information needed to load the Job result is sent from Matisse using UDP notification.

Finally, we also integrated data sharing trough WMS server. This enables to share the data trough any network with the only need of a client that can be a simple web browser.

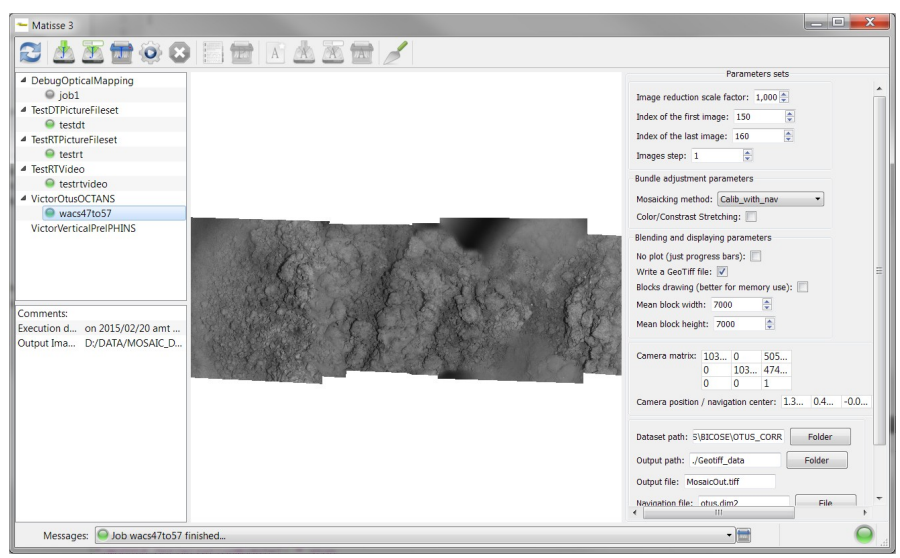

Fig. 6. Matisse mosaicking tool GUI seen from user view.

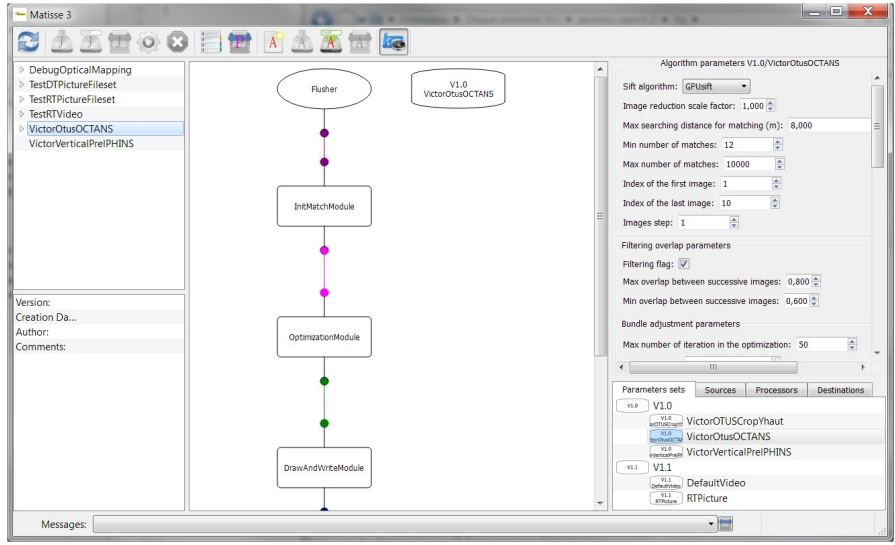

Fig. 7. Matisse mosaicking tool GUI seen from expert view.

\section{CONCLUSION}

Within the scope of our new HROV, we developped a complete optical mapping tool suite. This tool includes state-ofthe-art 2D and 3D mapping techniques adapted to operational needs. All Matisse algorithms have been evaluated on several datasets, at different scales, acquisition conditions, and camera types. This ensures the robustness of the proposed methods, so that Matisse can tend toward the objective to become an easy to use and mostly automatic mosaicking software, which can be used in a wide diversity of situations. Coupled with the high resolution images obtained with the HROV system, Matisse will improve the scientific analysis efficiency. 


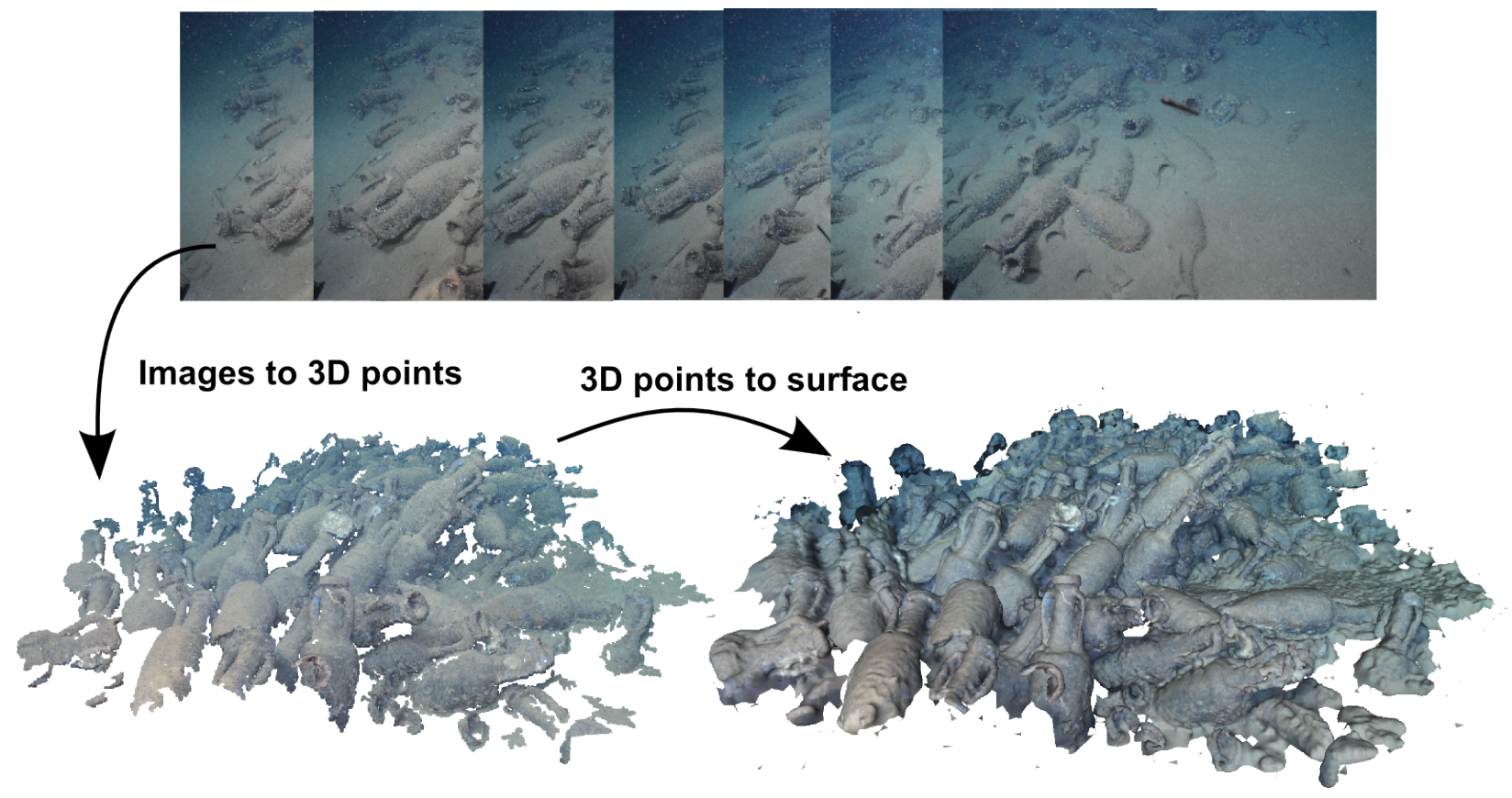

Fig. 5. This Figure schematically presents the 3D reconstruction steps. This example uses images obtained from a Nautile dive using the exact replica of the DSLR still camera mounted on the HROV. The DSLR high resolution (24Mpix) leads to a very dense 3D reconstruction.

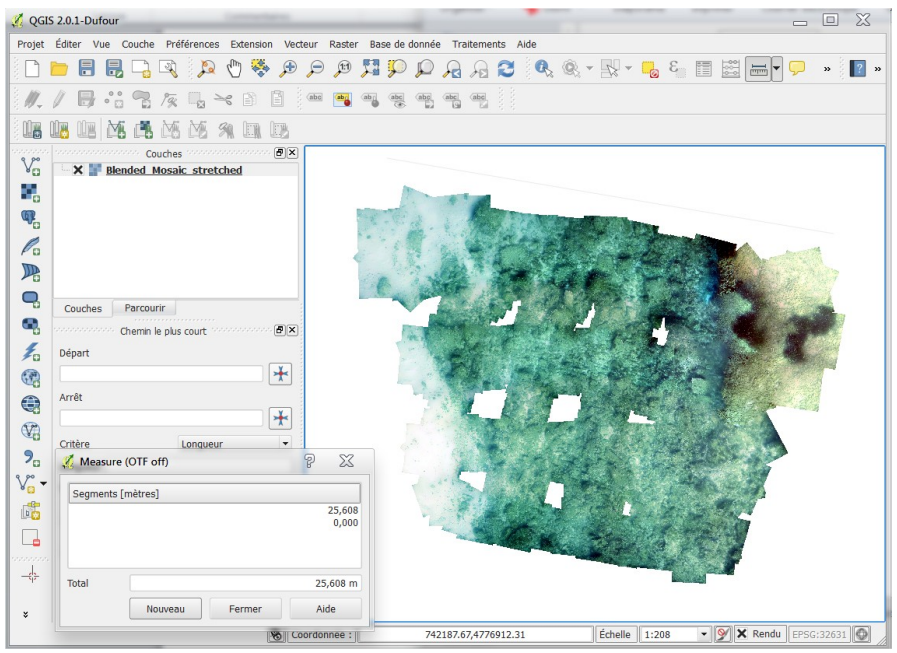

Fig. 8. QGIS provides a plug-in architecture. This figure presents an instance of QGIS which use our matisse plug-in to imports a processed mosaic with network communication.

\section{REFERENCES}

[1] O. Pizarro, H. Singh, 2003. Toward large-area mosaicking for underwater scientific applications. IEEE J Oceanic Eng, 28, 651-672.

[2] M. Borgetto, V. Rigaud, and J.F. Lots. Lighting correction for underwater mosaicking enhancement. Proceedings of the 16th International Conference on Vision Interface, Halifax,Canada, June 2003.

[3] J. Ferrer, A. Elibol, O. Delaunoy, N. Gracias, and R. Garcia, LargeArea Photo-Mosaics Using Global Alignment and Navigation Data., Proceedings of OCEANS 2007.

[4] F. Orsenigo, K. Sarantakos, Tornado: An Application for on-the-Fly Mosaicking through Phase Correlation. Proceedings of MCMC2009.
[5] H. Sakai, T. Tanaka, S. Ohata, M. Ishitsuka, K. Ishii, T. Ura,Applicability and Improvement of Underwater Video Mosaic System using AUV. Proceedings of OCEAN 2004.

[6] Vincent, A.G. and Pessel, N. and Borgetto, M. and Jouffroy, J. and Opderbecke, J. and Rigaud, V. Real-time geo-referenced video mosaicking with the MATISSE system. Proceedings of OCEAN 2003.

[7] V. Rigaud, J. Opderbecke, P. Simoni, and C. Pitout. Real time operational mosaics with the deep sea rov Victor. In Proceedings of the International Workshop on Underwater Robotics for Sea Exploitation and Environmental Monitoring - IARP 2001, Rio de Janeiro, Brasil,October 2001.

[8] A.-G. Allais, M. Borgetto, J. Opderbecke, N. Pessel, and V. Rigaud. Seabed video mosaicking with MATISSE : a technical overview and cruise results. In Proceedings of the (ISOPE Conference, pages 417421, Toulon, May 2004

[9] V. Brandou, A. G. Allais, M. Perrier, E. Malis, P. Rives, J. Sarrazin, and P. M. Sarradin, 3D Reconstruction of Natural Underwater Scenes Using the Stereovision System IRIS. Proceedings of OCEAN 2007.

[10] T. Nicosevici, N. Gracias, S. Negahdaripour, and R. Garcia, Efficient Three-Dimensional Scene Modeling and Mosaicing, Journal of Field Robotics 26(10), 759-788 (2009).

[11] N. Snavely, Scene Reconstruction and Visualization from Internet Photo Collections, $\mathrm{PhD}$ dissertation, 2008

[12] Josep Aulinas, Yvan R. Petillot, Xavier Llado, J. Salvi, Rafael Garcia Vision-Based Underwater SLAM for the SPARUS AUV, Proceedings of the 10th International Conference on Computer and IT Applications in the Maritime Industries

[13] F. Bruno, Gianfranco Bianco, Maurizio Muzzupappa, Sandro Barone, Armando Viviano Experimentation of structured light and stereo vision for underwater $3 D$ reconstruction, ISPRS Journal of Photogrammetry and Remote Sensing 2011

[14] Lowe, D.G. Object recognition from local scale-invariant features., Computer Vision, 1999. The Proceedings of the Seventh IEEE International Conference on (Volume:2).

[15] Burt, P., and Adelson, E. H., A Multiresolution Spline with Application to Image Mosaics. ACM Transactions on Graphics, 2(4):217-236, 1983.

[16] Vivek Kwatra, Arno Schdl, Irfan Essa, Greg Turk and Aaron Bobick. 
Graphcut Textures: Image and Video Synthesis Using Graph Cuts. in Proc. ACM Transactions on Graphics, SIGGRAPH 2003.

[17] Furukawa, Y.; Ponce, J., Accurate, Dense, and Robust Multiview Stereopsis, Pattern Analysis and Machine Intelligence, IEEE Transactions on , vol.32, no.8, pp.1362,1376, Aug. 2010

[18] Kazhdan, M.; Bolitho, M. and Hoppe, H. Poisson Surface Reconstruction, Eurographics Symposium on Geometry Processing 2006. 